\title{
Domain wall formation in late-time phase transitions
}

\author{
Edward W. Kolb
}

Institute for Theoretical Physics, University of California, Santa Barbara, CA 99106 and NASA/Fermilab Astrophysics C'enter

Fermi National Accelerator Laboratory, Batavia, IL 60510

and Department of Astronomy and Astrophysics, Enrico Fermi Institute The University of Chicago, Chicago, IL 60697

\section{Yun Wang}

Institute for Theoretical Physics, University of California, Santa Barbara, CA 99106 and Physics Depariment, University of Florida, Gainesville, FL 92611

\section{Abstract}

We investigate domain wall formation in late-time phase transitions. We find that as in the invisible-axion-domain-wall phenomenon, thermal efferts alone are insufficient to drive different regions of the Universe to different parts of the disconnected vacuum manifold. This suggests that domain walls do not form unless either there is some supplemental (but perhaps not unreasonable) dynamics to localize the scialar field responsible for the phase transition to the low-temperature maximum (to an extraordinary precision) before the onset of the phase transition, or there is some nonthermal mechanism to produce large fluctuations in the scalar field. The fact that domain wall production is not a robust prediction of late-time transitions may suggest future directions in model building.

\section{MASTER}




\section{INTRODUCTION}

There has recently been interest in late-time phase transitions ${ }^{1}$ as a possible solution to the large-scale structure problem. It has been proposed that the domain walls formed in a late-time (after decoupling) phase transition may seed the structure that we observe today, without conflicting with the well established isotropy of the microwave background radiation. (However, more detailed calculations show that this need not be the case. ${ }^{2}$ )

Previously, it has been taken for granted that domain walls with interesting sizes (100 kpc and larger) can form in a late-time phase transition. In this work, we apply the results of a previous investigation into the dynamics of late-time phase transitions ${ }^{3}$ to examine the dependence upon initial conditions of the scalar field for the scenario of wall formation. We find that the formation of domain walls is not a robust prediction of models with late-time phase transitions.

Before considering why dor ain walls might not form in a late-time phase transition, it is useful to recall why domain walls do form in standard phase transitions involving theorie" with disconnected vacuum manifolds. Consider a scenario for domain wall production in a simple model with a reflection symmetry $(\phi \leftrightarrow-\phi)$, described by a theory with a single scalar field $\phi$ and potential $V(\phi)=(\lambda / 4)\left(\phi^{2}-\sigma^{2}\right)^{2}$. The zerotemperature vacuum manifold has degenerate vacua $\langle\phi\rangle= \pm \sigma$. At temperatures above the critical temperature $T_{C}=2 \sigma$, the ground state of the system is $\langle\phi\rangle=0$. Furthermore, at temperatures much above the critical temperature, the (thermal) mass of the field, $m_{T}^{2}=\lambda T^{2} / 8$, increases with temperature. This has the effect of localizing the field about the high-temperature minimum. Below the critical temperature the high-temperature minimum becomes a local maximum and since the field is already localized about $\phi=0$, small thermal fluctuations will tend to push the field to one or 
the other low-temperature vacuum states. The direction of the thermal kick will be random on scales set by the thermal correlation length. Thus, regions of the Universe will settle in different vacuum states, establishing a domain-wall network.

We will refer to this picture of domain wall formation as the thermal mechanism, because it is thermal fluctuations that push the field to different vacua. We also note that thermal processes also play a role in positioning the field to a location where the thermal fluctuations can drive the field to different minima.

We will see that a (possibly crucial) ingredient missing in the late-time transition model we study is a mechanism to localize the field in a position where thermal fluctuations will kick the field to different vacua. This problem arises as a result of the peculiar thermal properties of the field involved in the transition, essentially due to the fact that the weak coupling of the field results in very small thermal fluctuations. This leads to a scenario where the field will not evolve to a position where thermal fluctuations, which will be small, will drive the field to different low-temperature vacua.

There are two ways around this problem. One could arrange for large fluctuations of super-horizon size in the scalar field well before the transition. In this case the wall network produced in the transition will reflect the initial field configuration, and there is no reason to expect the wall network to resemble the standard network as always assumed. A second possibility is to postulate that some dynamics, not part of the original model, acts to position the field correctly for the thermal fluctuations to operate. We will see that because the thermal fluctuations are so incredibly small, the positioning of the field must be done with great precision.

Our discussions are based on the particle physics models for late-time phase transition discussed in Ref. 3. The simplest model associated with phase transition consists of a single real scalar field $\phi$ with self interaction $\lambda \phi^{4}$. However, as pointed out in Ref. 3 , in the context of a late-time phase transition, the more reasonable models to 
consider are the ones in which phase transition is driven not through $\phi$ self interactions with the background, but rather by $\phi$ interactions with a background of some other field $\psi$, perhaps a fermion. The essence is to have $\phi-\psi$ interactions weak enough to provide a late-time, soft-wall transition, but $\psi$ can have additional interactions that can establish the background by thermal interactions.

This paper is divided into four parts. In Sec. II we calculate the thermal fluctuations of the scalar field involved in the transition. In Sec. III we consider the dynamics of the evolution of the scalar field in the early Universe. In the final section we conclude and discuss the prospects for wall production in late-time phase transitions.

\section{THERMAL FLUCTUATIONS IN SOF'T-WALL MODELS}

\section{A. YUKAWA MODEL}

First, let us consider the simple model of a single real scalar field $\phi$ interacting with a fermion $\psi$, with a potential:

$$
V_{0}(\phi, \psi)=-\frac{1}{2} m_{0}^{2} \phi^{2}+\frac{\lambda_{0}}{4} \phi^{4}-h \phi \bar{\psi} \psi
$$

where the parameters $m_{0}, \lambda_{0}$, and $h$ are the unrenormalized mass and coupling constants. Following the analysis of Ref. 3 , we assume that the fermion loops dorninate, which is the case if $h \gg \lambda_{0}$ and the boson tadpole is neglected. The renormaiized, one-loop effective potential at finite temperature is

$$
V_{1}(\phi)=-\frac{1}{16 \pi^{2}}(h \phi)^{4} \ln \frac{\phi^{2}}{\mu^{2}}+\Delta V_{T}(\phi)
$$

where $\mu$ is an arbitrary mass scale related to the values of coupling constants, and

$$
\Delta V_{T}(\phi)=-4 \frac{T^{4}}{2 \pi^{2}} \int_{0}^{\infty} d x x^{2} \ln \left[1+\exp \left(-\sqrt{x^{2}+m_{\psi}^{2} / T^{2}}\right)\right]
$$


with $m_{\psi} \equiv h \phi$.

At high temperature, $\Delta V_{T}(\phi) \simeq+h^{2} \phi^{2} T^{2} / 3$. Hence the curvature at $\phi=0$ (which is negative for $T=0$ ) will be positive for high $T$, and $\phi=0$ becomes the global minimum of the potential. Defining the critical temperature for the phase transition to be the temperature when $\partial^{2} V / \partial^{2} \phi$ vanishes at $\phi=0$, we have $T_{C} \sim h^{-1} m_{\phi}$. We want the phase transition to occur after recombination (but well before the present epoch) and to produce "soft" walls. These requirements lead to restrictions on $m_{\phi}\left(m_{\phi} \leqslant 10^{-24} \mathrm{eV}\right)$ and $T_{C}\left(\mathrm{eV} \gtrsim T_{C} \gtrsim 10^{-3} \mathrm{eV}\right)$. By making $h$ sufficiently small, it is possible to have $T_{C} \gg m_{\phi}$ for a late-time, soft-wall phase transition.

Thermal fluctuations in the field are found by examining the finite-temperature, two-point correlation function. The two-point correlation function is given by the Green's function

$$
\langle\phi(x) \phi(0)\rangle_{T}=G_{T}(x)=\int \frac{d^{4} k}{(2 \pi)^{4}} e^{-i k x} D_{T}(k),
$$

where $D_{T}(k)$ is the $\phi$ propagator at finite temperature. In keeping with the assumption that $\phi$ is very weakly coupled, we will assume that it is not in equilibrium and there is no thermal $\phi$ background. Thus the thermal effects enter only through the interactions of $\phi$ with the thermal background of $\psi$ s. To compute $D_{T}(k)$, consider the one-loop diagram in Fig. 1. Recall that the fermion propagator at finite temperature is ${ }^{4}$

$$
S_{T}(p)=i\left(\not p-m_{\psi}\right)^{-1}-2 \pi f_{\psi}\left(E_{p}\right)\left(\not p+m_{\psi}\right) \delta\left(p^{2}-m_{\psi}^{2}\right),
$$

where $f_{\psi}$ is the phase space density for $\psi$. Let us assume that the phase space density for $\psi$ is that of a thermal distribution (i.e., a Fermi-Dirac distribution with temperature $T)$. With this we have

$$
D_{T}(k)=i\left(k^{2}-m_{\phi}^{2}\right)^{-1}+i\left(k^{2}-m_{\phi}^{2}\right)^{-1} \cdot \text { loop } \cdot i\left(k^{2}-m_{\phi}^{2}\right)^{-1},
$$

where 


$$
\text { loop } \equiv(-1) \int \frac{d^{4} p}{(2 \pi)^{4}} \operatorname{Tr}\left\{i h S_{T}(p) i h S_{T}(p-k)\right\}
$$

We will ignore the temperature-independent contribution of the loop to $D_{T}(k)$, as it is absorbed in the renormalization of the $\phi$ propagator, and concentrate on the temperature-dependent contribution. Denoting the temperature-dependent part of the loop as loop , we have

$$
\begin{aligned}
\operatorname{loop}_{T}= & \frac{4 h^{2}}{(2 \pi)^{4}} \int d^{4} p\left[p(p-k)+m_{\psi}^{2}\right]\left\{-i 4 \pi \frac{f_{\psi}\left(E_{p}\right)}{(p-k)^{2}-m_{\psi}^{2}} \delta\left(p^{2}-m_{\psi}^{2}\right)\right. \\
& \left.+4 \pi^{2} f_{\psi}\left(E_{p}\right) f_{\psi}\left(E_{p-k}\right) \delta\left(p^{2}-m_{\psi}^{2}\right) \delta\left((p-k)^{2}-m_{\psi}^{2}\right)\right\} .
\end{aligned}
$$

The leading term in this expression corresponds to taking the thermal part of one fermion propagator and the temperature-independent part for the other, i.e., the first term. The temperature-dependent two-point correlation function is then given by

$$
\begin{aligned}
G_{T}(x)= & i \frac{8 h^{2}}{(2 \pi)^{3}} \int d^{4} p f_{\psi}\left(E_{p}\right) \delta\left(p^{2}-m_{\psi}^{2}\right) \\
& \times \int \frac{d^{4} k}{(2 \pi)^{4}} e^{-i k x} \frac{p(p-k)+m_{\psi}^{2}}{\left(k^{2}-m_{\phi}^{2}\right)^{2}\left[(k-p)^{2}-m_{\psi}^{2}\right]} .
\end{aligned}
$$

Now at finite temperature there are three length scales in the problem that appear in $G_{T}(x): m_{\phi}^{-1}, m_{\psi}^{-1}$, and $T^{-1}$. It is useful to define the following dimensionless length scales:

$$
\rho_{\phi} \equiv|\mathbf{x}| / m_{\phi}^{-1} ; \quad \rho_{\psi} \equiv|\mathbf{x}| / m_{\psi}^{-1} ; \quad \rho_{T} \equiv|\mathbf{x}| / T^{-1} ; \quad z \equiv \rho_{\psi} / \rho_{T}
$$

After much manipulation, we may express $G_{T}(x)$ in terms of sums and integrals involving modified Bessel functions:

$$
\begin{aligned}
G_{T}(|\mathbf{x}|) \simeq & \frac{2 h^{2} m_{\psi}^{2}}{(2 \pi)^{4}} \sum_{n=1}^{\infty}(-1)^{n+1}\left\{z ^ { - 1 } \left[-\frac{K_{1}(z n)}{n} K_{0}\left(\rho_{\phi}\right)\right.\right. \\
& \left.+\int_{0}^{1} d \theta \frac{K_{1}\left(z \sqrt{n^{2}+(1-\theta)^{2} \rho_{T}^{2}}\right)}{\sqrt{n^{2}+(1-\theta)^{2} \rho_{T}^{2}}} K_{0}\left(\sqrt{(1-\theta)^{2} \rho_{\psi}^{2}+\theta \rho_{\phi}^{2}}\right)\right]
\end{aligned}
$$




$$
\begin{gathered}
+2\left(\rho_{T}\right) \int_{0}^{1} d \theta \theta \frac{K_{1}\left(z \sqrt{n^{2}+(1-\theta)^{2} \rho_{T}^{2}}\right)}{\sqrt{n^{2}+(1-\theta)^{2} \rho_{T}^{2}}} \\
\left.\times \frac{K_{1}\left(\sqrt{(1-\theta)^{2} \rho_{\psi}^{2}+\theta \rho_{\phi}^{2}}\right)}{\sqrt{(1-\theta)^{2}+\theta m_{\phi}^{2} / m_{\psi}^{2}}}\right\},
\end{gathered}
$$

where we have used $m_{\phi} \ll m_{\psi}$, which will be true in the context of late-time phase transitions. Note that for $|\mathbf{x}|=0$, we have

$$
G_{T}(0)=\frac{h^{2}}{(2 \pi)^{3}} \frac{m_{\psi}^{2} T}{m_{\phi}} \sum_{n=1}^{\infty} \frac{(-1)^{n+1}}{n} K_{1}(n z)
$$

which for $T \gg m_{\psi}$ gives $G_{T}(0)=\left(h^{2} / 96 \pi\right)\left(m_{\psi} / m_{\phi}\right) T^{2}$, the familiar $T^{2}$ dependence of thermal fluctuations in $\phi^{2}$.

For $|\mathbf{x}| \neq 0$, we are able to obtain an upper limit for $G_{T}(|\mathbf{x}|)$ :

$$
G_{T}(|\mathbf{x}|) \leq \frac{\pi h^{2}}{(2 \pi)^{4}} \frac{m_{\psi}^{2} T}{m_{\phi}} K_{1}\left(\rho_{\phi}\right) \ln \left(1+e^{-z}\right),
$$

again assuming that $m_{\phi} \ll m_{\psi}$.

For the analysis of domain wall formation, we will be interested in correlations in $\phi$ on length scales comparable to the scale of the correlation length in the transition, $|\mathbf{x}| \sim m_{\phi}^{-1}$, i.e., $\rho_{\phi} \sim 1$. Since $T_{C} \sim m_{\phi} / h$, and $T_{C} \leq 0.1 \mathrm{eV} \sim m_{\psi}$ as discussed previously, it follows that that $h \sim m_{\phi} / m_{\psi}$. Hence

$$
G_{T}\left(\rho_{\phi} \simeq 1\right) \leq m_{\phi} T K_{1}\left(\rho_{\phi}\right)
$$

Since $\rho_{\phi} \ll \rho_{\psi} \lesssim \rho_{T}$ for late-time phase transitions, it is no surprise that the relevant dimensionless length scale is $\rho_{\phi}$. Note that $K_{1}\left(\rho_{\phi} \sim 1\right)$ is of order unity, while $K_{1}\left(\rho_{\phi} \gg\right.$ 1) $\sim \sqrt{\pi / 2 \rho_{\phi}} \exp \left(-\rho_{\phi}\right)$. It is clear that the correlations in $\phi$ are rather insubstantial on scales relevant to domain wall formation since $m_{\phi} \ll T_{C} \sim m_{\psi}$.

This simple model for late-time phase transition involves a very small, perhaps unnatural dimensionless coupling constant $h$. In the next section we study a more 
realistic model in which there is physical motivation for the origin of small coupling constants.

\section{B. CHIRAL MODELS}

Let us now consider a theory with the Lagrangian proposed by Hill and Ross: ${ }^{5}$

$$
\begin{aligned}
\mathcal{L}= & \frac{1}{2} \partial^{\mu} \phi \partial_{\mu} \phi+\bar{\nu}_{L} i \not \partial \nu_{L}+\bar{\nu}_{R} i \not \partial \nu_{R}+\left(m \bar{\nu}_{L} \nu_{R} e^{i \phi / f}+\epsilon \bar{\nu}_{L} \nu_{R}+\text { h.c. }\right) \\
& +\widetilde{m}^{4} \cos \left(\frac{\phi}{f}+\delta\right) .
\end{aligned}
$$

Note that the above theory has non-linearly realized chiral $U(1)$ symmetry when the $\epsilon$-term and the $\widetilde{m}$-term are not present. The $\widetilde{m}$-term has been added to give $\phi$ a mass of unspecified origin, this usually comes from some deeper (compared to the spontaneous breaking of the original chiral $U(1)$ symmetry) symmetry breaking in the theory which breaks the continuous $U(1)$ down to a discrete subgroup $\mathcal{Z}_{N}$. The $\epsilon$-term can be interpreted as the additional manifestation of this deeper symmetry breaking, similar to the $\sigma$-term in the nucleon-pion system. It breaks the chiral symmetry explicitly.

Since a nonzero $\widetilde{m}$ term will always be induced by the presence of a nonzero $\epsilon$ and $m$, let us write down the following Lagrangian which contains $N$ Dirac neutrino species and is invariant under a $\mathcal{Z}_{N}$ discrete symmetry:

$$
\mathcal{L}=\frac{1}{2} \partial^{\mu} \phi \partial_{\mu} \phi+\sum_{j=0}^{N-1} \bar{\nu}_{j} i \not \partial \nu_{j}+\sum_{j=0}^{N-1}\left(m+\epsilon e^{i \phi / f+2 i j \pi / N}\right) \bar{\nu}_{j L} \nu_{j R}+h . c .
$$

First consider the $N=2$ case. The effective low-energy theory (energy scales much less than $f$ ) has Yukawa couplings of the form:

$$
\begin{aligned}
\mathcal{L}_{Y U K} & =M_{+}(\phi) \bar{\psi}_{0} \psi_{0}+M_{-}(\phi) \bar{\psi}_{1} \psi_{1} \\
M_{ \pm}^{2}(\phi) & =m^{2}+\varepsilon^{2} \pm 2 m \epsilon \cos (\phi / f)
\end{aligned}
$$

It has been shown in Ref. (3) that the finite temperature potential for $\phi$ in the $\mathcal{Z}_{2}$ 
case is

$$
\begin{aligned}
\frac{V_{T}(\phi)}{m_{-}^{4}}= & \frac{1}{32 \pi^{2}}\left(1+r^{2}\right)^{2} \ln \left(\frac{r^{2}+1}{2}\right) \\
+ & {\left[-\frac{1}{2} \frac{f^{2} m_{0}^{2}}{m_{-}^{4}}+\frac{\left(r^{2}-1\right)}{32 \pi^{2}}\left(r^{2} \ln r^{2}+\frac{r^{2}-1}{2}\right)\right] \cos ^{2}\left(\frac{\phi}{f}\right) } \\
& -\frac{1}{16 \pi^{2}}\left[\left(\frac{M_{+}(\phi)}{m_{-}}\right)^{4} \ln \frac{M_{+}^{2}(\phi)}{m_{-}^{2}}+\left(\frac{M_{-}(\phi)}{m_{-}}\right)^{4} \ln \frac{M_{-}^{2}(\phi)}{m_{-}^{2}}\right] \\
& -\frac{4}{2 \pi^{2}}\left(\frac{T}{m_{-}}\right)^{4} \sum_{j=+--} \int_{0}^{\infty} d x x^{2} \ln \left[1+\exp \left(-\sqrt{x^{2}+M_{j}^{2}(\phi) / T^{2}}\right)\right]
\end{aligned}
$$

where we have defined

$$
m_{ \pm} \equiv m \pm \epsilon ; \quad r \equiv m_{+} / m_{-} ; \quad m_{0}^{2} \equiv V^{\prime \prime}(\phi=0) .
$$

The corresponding temperature-dependent mass is defined as $m_{\phi}^{2}(T) \equiv V_{T}^{\prime \prime}(\phi=0)$ :

$$
\begin{aligned}
m_{\phi}^{2}(T) & =m_{0}^{2}+\frac{2 m \epsilon T^{2}}{\pi^{2} f^{2}} \sum_{j=+,-}(-j) \int_{0}^{\infty} d x x^{2} \frac{\left(x^{2}+m_{j}^{2} / T^{2}\right)^{-1 / 2}}{1+\exp \left(x^{2}+m_{j}^{2} / T^{2}\right)^{1 / 2}} \\
& =m_{0}^{2}+\frac{2 m \epsilon T}{\pi^{2} f^{2}} \sum_{n=1}^{\infty} \frac{(-1)^{n+1}}{n}\left[m_{-} K_{1}\left(n \frac{m_{-}}{T}\right)-m_{+} K_{1}\left(n \frac{m_{+}}{T}\right)\right] .
\end{aligned}
$$

For $T \gg m_{ \pm}$, we get

$$
m_{\phi}^{2}(T)=m_{0}^{2}+\frac{m \epsilon}{\pi^{2} f^{2}}\left[m_{+}^{2} \ln \frac{T}{m_{+}}-m_{-}^{2} \ln \frac{T}{m_{-}}\right] .
$$

Obviously, at high $T$, we have $m_{\phi}^{2}(T)>0$, i.e., we have a phase transition with $T_{C}$ satisfying $m_{\phi}^{2}\left(T_{C}\right)=0$, provided that $m_{0}^{2}<0$.

By expanding the mass functions $M_{ \pm}(\phi)$ in Eq. (2.17), we find the lowest-order coupling of $\phi$ to the fermions:

$$
\begin{aligned}
& \mathcal{L}_{i}=h_{+} \phi \phi \bar{\psi}_{0} \psi_{0}+h_{-} \phi \phi \bar{\psi}_{1} \psi_{1} \\
& h_{ \pm}=\mp \frac{1}{m_{ \pm}} \frac{m \epsilon}{f^{2}} .
\end{aligned}
$$

To compute the two-point function, we need the lowest-order correction to the $\phi$ propagator, see Fig. 2. The correlation function is still given by Eqs. (2.4) and (2.6), with 


$$
\text { loop }=(-1) \int \frac{d^{4} p}{(2 \pi)^{4}} \operatorname{Tr}\left(i h_{+} S_{T}^{+}(p)+i h_{-} S_{T}^{-}(p)\right)
$$

where

$$
S_{T}^{ \pm}=i\left(\not p-m_{ \pm}\right)^{-1}-2 \pi f_{\psi}\left(E_{p}\right)\left(\not p+m_{ \pm}\right) \delta\left(p^{2}-m_{ \pm}^{2}\right)
$$

Let the temperature-dependent part of loop be loop . We then have

$$
\begin{aligned}
\operatorname{loop}_{T} & =\operatorname{loop}_{T}^{+}+\operatorname{loop}_{T}^{-} \\
\operatorname{loo} p_{T}^{ \pm} & =\frac{i}{(2 \pi)^{2}} h_{ \pm} m_{ \pm}^{2} T \sum_{n=1}^{\infty} \frac{(-1)^{n+1}}{n} K_{1}\left(n \frac{m_{ \pm}}{T}\right) .
\end{aligned}
$$

Eq. (2.4) now gives

$$
G_{T}(|\mathbf{x}|)=-\frac{i}{2(2 \pi)^{2}} K_{0}\left(\rho_{\phi}\right) \cdot \operatorname{loop}_{T}
$$

Hence we obtain

$$
\begin{aligned}
G_{T}(|\mathbf{x}|)= & \frac{m \epsilon T}{2(2 \pi)^{4} f^{2}} K_{0}\left(\rho_{\phi}\right) \\
& \times \sum_{n=1}^{\infty} \frac{(-1)^{n+1}}{n}\left[m_{-} K_{1}\left(n \frac{m_{-}}{T}\right)-m_{+} K_{1}\left(n \frac{m_{+}}{T}\right)\right] .
\end{aligned}
$$

For $T \gg m_{ \pm}$, we have

$$
G_{T}(|\mathbf{x}|) \simeq \frac{1}{(2 \pi)^{4}}\left(\frac{m \epsilon}{f}\right)^{2} K_{0}\left(\rho_{\phi}\right) \ln (T / m) .
$$

Note that $G_{T}(|\mathbf{x}|)$ is singular for $|\mathbf{x}| \rightarrow 0$, which is natural since the effective Lagrangian of Eq. (2.15) is not renormalizable.

$G_{T}(|\mathbf{x}|)$ is well behaved for $|\mathbf{x}| \neq 0$ and gives us the correlation function between two spatially separated points in the $\phi$ field. Note that

$$
K_{0}\left(\rho_{\phi} \gg 1\right) \sim \sqrt{\frac{\pi}{2 \rho_{\phi}}} \exp \left(-\rho_{\phi}\right),
$$

\section{DISCLAIMER}

This report was prepared as an account of work sponsored by an agency of the United States Government. Neither the United States Government nor any agency thereof, nor any of their employees, makes any warranty, express or implied, or assumes any legal liability or responsibility for the accuracy, completeness, or usefulness of any information, apparatus, product, or process disclosed, or represents that its use would not infringe privately owned rights. Reference herein to any specific commercial product, process, or service by trade name, trademark, manufacturer, or otherwise does not necessarily constitute or imply its endorsement, recommendation, or favoring by the United States Government or any agency thereof. The views and opinions of authors expressed herein do not necessarily state or reflect those of the United States Government or any agency thereof. 
while $K_{0}\left(\rho_{\phi} \sim 1\right)<1$. Obviously, the correlations in $\phi$ field are suppressed severely on the scale of $|\mathbf{x}| \sim m_{\phi}^{-1}$, since $\epsilon \lesssim m \ll f$.

In soft-boson particle physics models associated with late-time phase transitions we have the typical scales $m_{\psi} \sim 0.1 \mathrm{eV} ; f \sim 10^{15} \mathrm{GeV} ; T_{C} \sim m_{\psi}$; and $m_{\phi} \sim m_{\psi}^{2} / f$. The domain walls are cosmologically interesting because the walls have scale $m_{\phi}^{-1} \sim$ $m_{\psi}^{-1}\left(f / m_{\psi}\right)$. The smallness of $\left(m_{\psi} / f\right)$ is essential in making the wall scale large enough to be of interest to large-scale structure, however, it also leads to suppression of the correlation in the $\phi$ field due to thermal effects. Stochastic noise is washed out in the large volume $m_{\phi}^{-3}$.

\section{THE EVOLUTION OF $\phi$}

In the last section we demonstrated that a generic feature of the late-time, softwall models is very small thermal noise in the system. This means if wall formation follows the standard scenario, there must be some mechanism to poise the field at a lowtemperature maximum to an extraordinarily high precision, one part in $(\mathrm{f} / \mathrm{m})^{2} \simeq 10^{50}$. Is this reasonable? Well, since the low-temperature maximum is a high-temperature minimum, the field naturally will evolve toward the desired point and undergo damped oscillation about that point. In this section we show that, as one might expect, the oscillations will not be damped enough to set the field to the desired point to the necessary precision.

Let us now turn to the evolution of the $\phi$ field in the potential $V_{T}(\phi)$ given by Eq. (2.18). In Fig. 3, $V_{T}(\phi)$ is plotted for several different temperatures. The phase transition occurs when the potential turns over at $T=T_{C}$. Solving Eq. (2.20) numerically with $m_{\phi}^{2}(T)=0$, we obtain $T_{C}$ as a function of $\left(-m_{0}^{2} f^{2} / m_{-}^{4}\right)$, given $r \equiv m_{+} / m_{-}$. 
The result is plotted in Fig. 4. Note that the high temperature behaviour of the $T_{C}$ curves agrees with the formula obtained from Eq. $(2.21):^{3}$

$$
T_{C}=m_{+}\left(\frac{m_{+}}{m_{-}}\right)^{m_{-}^{2} /\left(m_{+}^{2}-m_{-}^{2}\right)} \exp \left(\frac{8 \pi^{2} f^{2}\left(-m_{0}^{2}\right)}{\left(m_{+}^{2}-m_{-}^{2}\right)^{2}}\right) .
$$

The issue at hand is if the "initial" value of $\phi, \phi\left(T \gg T_{C}\right)$ is displaced from the hightemperature minimum, will it relax sufficiently close to the high-temperature minimum by $T_{C}$ so that it can receive random thermal kicks to different regions of the lowtemperature vacuum manifold. To address this issue we follow the equation of motion for the scalar field:

$$
\begin{aligned}
& \ddot{\phi}+3 H \dot{\phi}+\frac{\partial V_{T}}{\partial \phi}=0 \\
& H^{2}=\frac{8 \pi G_{N}}{3} \frac{\pi^{2}}{30} g_{*} T^{4}
\end{aligned}
$$

where we have assumed a radiation-dominated Universe.

Using the dimensionless quantities

$$
\theta \equiv \frac{\phi}{f} ; \quad \bar{T} \equiv \frac{T}{m_{-}} ; \quad v_{T}(\theta) \equiv \frac{V_{T}(\phi)}{m_{-}^{4}} ; \quad \bar{f} \equiv \frac{-m_{0}^{2} f^{2}}{m_{-}^{4}}
$$

after some manipulation, the equation of motion becomes

$$
\frac{d^{2} \theta}{d \bar{T}^{2}}=-\frac{90}{8 \pi^{3} g_{*}}\left(\frac{M_{P L}}{f}\right)^{2} \frac{1}{\bar{T}^{6}} \frac{\partial v_{T}}{\partial \theta} .
$$

There is no analytical solution to this equation. A numerical solution is shown by the solid curve of Fig. 5. As expected, the $\phi$ field oscillates about the high-temperature minimum (here $\phi=0$ ), then after the phase transition it evolves to a low-temperature minimum (here $\phi=-\pi / 2$ ). The reason the field evolved to $\phi=-\pi / 2$ rather than to the degenerate low-temperature minimum $\phi=+\pi / 2$ is simply because at the temperature of the phase transition the field was negative in its oscillations about $\phi=0$.

In order for walls to form we want some mechanism to send the field to $\phi=-\pi / 2$ in some regions and $\phi=+\pi / 2$ in other regions. Now if we wish we can model the thermal 
noise in the system by the well studied stochastic approach. ${ }^{6}$ However for thermal fluctuations to push $\phi$ to different regions, they must have an amplitude comparable to the oscillation amplitude. We saw from the previous section that the amplitude of thermal fluctuations are very small indeed. Is there any way for the amplitude of oscillations about $\phi=0$ to be damped sufficiently to empower thermal fluctuations? Clearly for the parameters of Fig. 5 the onswer is no.

We can explore the sensitivity of the oscillation amplitude at $T_{C}$ upon model patameters by considering the adiabatic invariant of the oscillating system. ${ }^{7}$

For oscillations near $\theta=0$

$$
\frac{\partial v_{T}}{\partial \theta}=\frac{f}{m_{-}^{4}} \frac{\partial V_{T}}{\partial \phi}=\frac{f^{2}}{m_{-}^{4}} m_{\phi}^{2}(T) \theta
$$

and we have $d^{2} \theta / d \vec{T}^{2}=-\lambda^{2} \theta$, with

$$
\lambda \equiv \sqrt{\frac{90}{8 \pi^{3} g_{*}}}\left(\frac{M_{P L}}{m_{-}^{2}}\right) \frac{m_{\phi}(T)}{\bar{T}^{3}} .
$$

If $\lambda$ were constant, the systern would be closed and would execute a strictly periodic motion with a constant energy $E$ and a fixed period $\lambda^{-1}$, i.e., $\theta=A \cos (\lambda \bar{T})$. When $\lambda$ is variable, the system is not closed and its energy not conserved. If $\lambda$ changes slowly, the rate of change in $E$ will be small. The dependence of $E$ on $\lambda$ can be expressed as the adiabatic invariant $I=E / \lambda \simeq \lambda A^{2}$, when the adiabatic condition $\left|\lambda^{-1} d \lambda / d \bar{T}\right| \ll \lambda$ is satisfied.

The $\phi$ field first begins to iscillate at $t \sim m_{\phi}^{-1}$. Since $T^{2}=\sqrt{90 / 8 \pi^{3} g_{*}}\left(M_{P L} / 2 t\right)$,

$$
\bar{T}_{\text {orc }} \equiv \bar{T}\left(t=m_{\phi}^{-1}\right)=\left(\frac{1}{2} \sqrt{\frac{90}{8 \pi^{3} g_{m}}}\right)^{1 / 2} \sqrt{\frac{m_{\phi} M_{P L}}{m_{-}^{2}}} \sim \mathcal{O}(1) \sqrt{\frac{M_{P L}}{\pi^{2} f}} .
$$

It is straightforward to show that at $T_{\text {osc }}$ we can use the adiabatic approximation. Of course at $T=T_{C}$, the adiabatic condition breaks down, because $m_{\phi}\left(T_{C}\right)=0$ leads to $\lambda\left(T_{C}\right)=0$. If we are only interested in a lower linit to the amplitude of the 
oscillation, we may set $m_{\phi}$ equal to a constant, say its zero-temperature value. In this case $A \propto \lambda^{-1 / 2} \sim T^{3 / 2}$, and $A\left(T_{C}<T<T_{\text {osc }}\right) \simeq A\left(T_{\text {osc }}\right) \times\left(T / T_{\text {osc }}\right)^{3 / 2}$.

Now we can compute the amplitude of the classical oscillations near $\theta=\phi / f=0$ for $T$ close to $T_{C}$ (assuming $T_{C}<T_{\text {osc }}$; if the inequality is not obeyed, there is no damping):

$$
A\left(T \simeq T_{C}\right) \simeq A\left(T=T_{\text {osc }}\right)\left(\frac{T_{C}}{T_{\text {osc }}}\right)^{3 / 2},
$$

and use Eq. (3.1) for $T_{C}$ and Eq. (3.7) for $T_{\text {osc }}$.

An example of this approximation is shown in Fig. 5. The solid curve is a result of a numerical integration of the equation of motion, and the dashed curve is the adiabatic approximation for the oscillation amplitude. The parameters chosen for the figure are $f / M_{P L}=10^{-4}, \bar{f}=1, m_{+}=3 m_{-}$, and $\phi\left(T \gg T_{C}\right) / f=1$. We find that the adiabatic approximation holds for the range of parameters of interest. For the entire parameter space the amplitude of sscillation is never reduced by more than a few orders of magnitude.

The conclusions are clear: There are insufficient oscillations brtween $T_{\text {osc }}$ and $T_{C}$ to damp the amplitude of the oscillations to the desired scale of [see Eq. (2.28)]

$$
\left\langle\phi^{2}\right\rangle_{T}^{1 / 2}=\sqrt{\left\langle\phi(0) \phi\left(\rho_{\phi} \sim 1\right)\right\rangle_{T}}=\sqrt{G_{T}(|\mathbf{x}|)} \simeq \frac{1}{(2 \pi)^{2}}\left(\frac{m \epsilon}{f}\right) \sqrt{\ln \bar{T}}
$$

which would correspond to $A\left(T_{C}\right) \sim m \epsilon / f^{2} \sim 10^{-50}$.

Since thermal effects can not fin $\phi$ to a value where thermal effects can kick the $\phi$ field to different minima, $\phi$ has to be initially localized (to extreme accuracy) to $\phi=0$ via some non-thermal mechanism for domain walls to form via the thermal process.

Finally, we mention that although we have only studied the $\mathcal{Z}_{2}$ model, our conclusions also hold for $\mathcal{Z}_{N>2}$ models as well. In fact, for these models the high-temperature $\phi$ mass is driven to zero, so there is even less of a force driving $\phi$ to the appropriate position. 


\section{CONCLUSIONS AND PROSPECTUS}

We have studied the thermal effects on the two-point $\phi$ correlation functions for two models associated with late-time phase transition. The first model is the simple $\lambda \phi^{4}$ theory with Yukawa coupling between $\phi$ and some thermal fermion $\psi$, the second model is more natural and involves a cosine potential for $\phi$ induced by its coupling to thermal neutrinos $\psi_{0}$ and $\psi_{1}$. We have shown that in both cases the thermal effects are suppressed due to the smallness of $m_{\phi}$ compared to $m_{\psi}$. Since the essential feature of a late-time phase transition model is to have domain walls with cosmologically large scales cqused by the smallness of $m_{\phi}$, we conclude that domain wall formation does not foilow the usual thermal scenario unless there is non-thermal mechanism for localizing $\phi$ to zero initially.

Of cousse one might imagine that the formation of domain walls in late-time phase transitions does not follow the thermal scenario, but results simply because long before $T_{C}$ inere are large, non-thermal fluctuations of the field, and the classical evolution of the field takes the field to different regions of the vacuum manifold. In some sense, the walls are present as initial conditions at $T_{C}$. If one adopts this approach, a domain wall network will result with characteristics (wall sizes, distributions, velocities, etc.) that reflect the initial conditions, and need not resemble the properties of walls produced by the thermal process. One must be careful to arrange these fluctutions before inflation, since inflation will erase them on scales of interest.

This non-thermal scenario is far from impossible, since the starting Lagrangian is of course only a low-energy approximation to the complete theory. It may well turn out to be natural that the seemingly highly contrived conditions necessary for domain wall formation are a natural result of the ultimate theory. Until such models are constructed, it is impossible to make predictions sensitive to the domain wall network, 
since the network depends upon initial conditions.

Our work suggests that if late-time phase transitions play a role in structure formation, it is more likely to be through slow-roll scenarios like those studied by Frieman, Hill, and Watkins ${ }^{8}$ than through formation of domain walls.

\section{Acknowledgements}

The work was supported in part by the Department of Energy and NASA (grant \#NAGW-2831) at Fermilab, and Department of Energy grant \#DE-FG05-86ER40272 at University of Florida, and the NSF (grant \#PHY89-04035) at the Institute for Theoretical Physics. Y. W. thanks the Fermilab Astrophysics Group for hospitality when part of this work was dune. We would like to thank Chris Hill for many useful discussions.

\section{References}

1. C. T. Hill, D. N. Schramm, and J. Fry, Comm. on Nucl. and Part. Phys. 19, 25 (1989).

2. M. S. Turner and A. Stebbins, Astrophys. J. Lett. 339, L13 (1989); M. S. Turner, R. Watkins, and L. M. Widrow, Astrophys. J. Lett. 367, L43 (1991).

3. A. K. Gupta, C. T. Hill, R. Holman, and E. W. Kolb, Phys. Rev. D 45, 441 (1992).

4. L. Dolan and R. Jackiw, Phys. Rev. D9, 3320 (1974).

5. C. T. Hill and G. G. Ross, Nucl. Phys. B311, 253 (1988); Phys. Lett. 203B, 125 (1988).

6. For example, see H. M. Hodges, Phys. Rev. D 39, 3557 (1989). 
7. L. D. Landau and E. M. Lifshitz, in Mechanics, (Pergamon Press, London, 1976).

8. J. A. Frieman, C. T. Hill, and R. Watkins, Fermilab report FNAL-91/324-A. 


\section{FIGURE CAPTIONS}

Figure 1. One-loop diagram of the $\phi$ propagator for the model with simple Yukawa couplings.

Figure 2. One-loop diagram of $\phi$ propagator for the $\mathcal{Z}_{2}$ soft-boson model.

Figure 3. The total potential $V_{T}(\phi)$ for the $\mathcal{Z}_{2}$ model, plotted for several different temperatures. The phase transition occurs when the potential turns over at $T=T_{C}$.

Figure 4. Numerical solution to $m_{\phi}^{2}\left(T_{C}\right)=0 . T_{C}$ is plotted as a function of $\left(-m_{0}^{2} f^{2} / m_{-}^{4}\right)$, for several different values of $r \equiv m_{+} / m_{\ldots}$. Read from top to bottom, the curves are for $r=1.01,3.05,1.5,2,3$, and 5 , respectively.

Figure 5. Example of the evolution of the $\phi$ field. The solid curve is a result of numerical integration of the equation of motion, and the dashed line is the adiabatic approximation for the oscillation amplitude. Parameters for the model are $f / M_{P L}=$ $10^{-4}, \bar{f}=1, m_{+}=3 m_{-}$, and $\phi\left(T \gg T_{C}\right) / f=1$. 


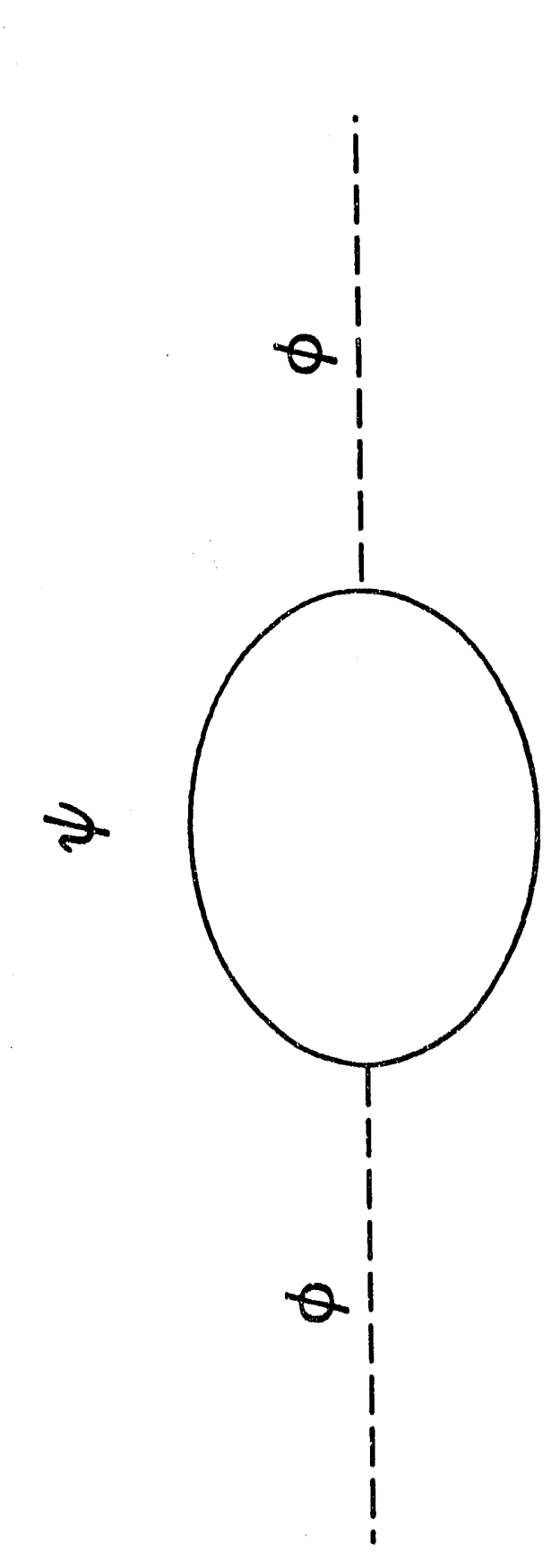

家

3
3
3
3
3 


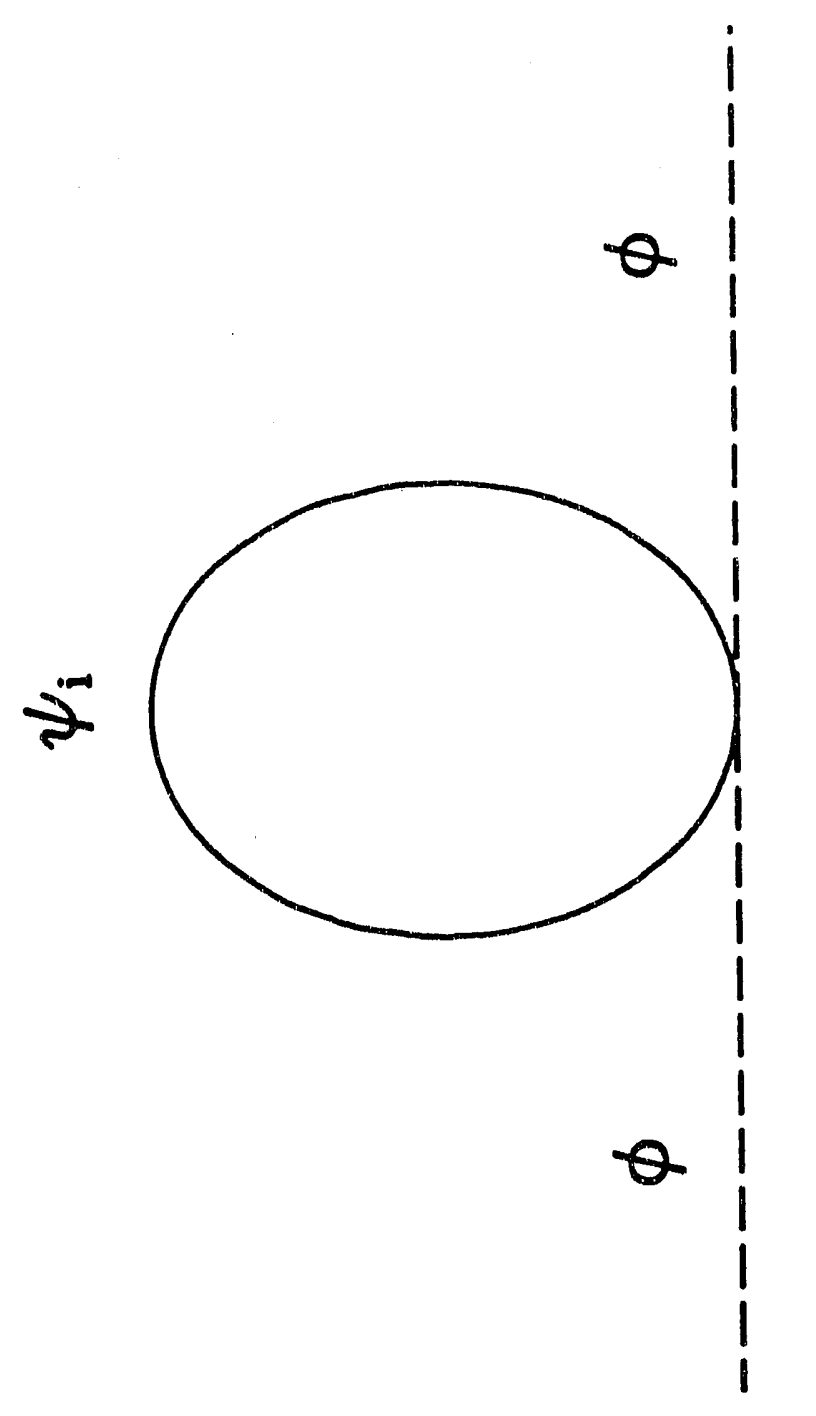

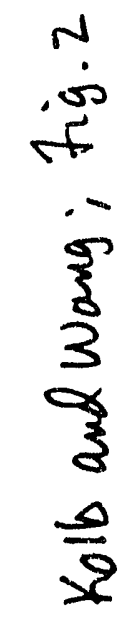




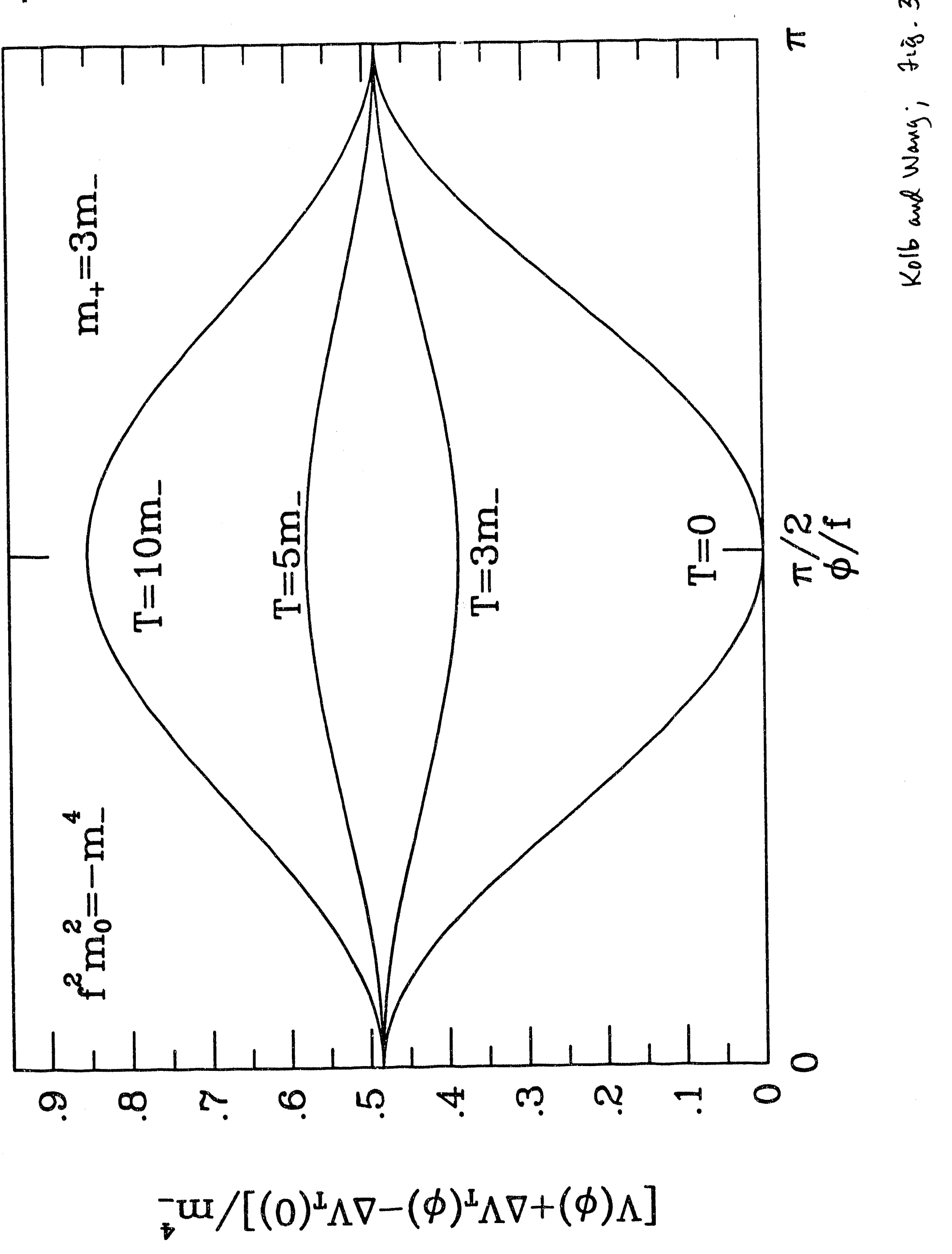




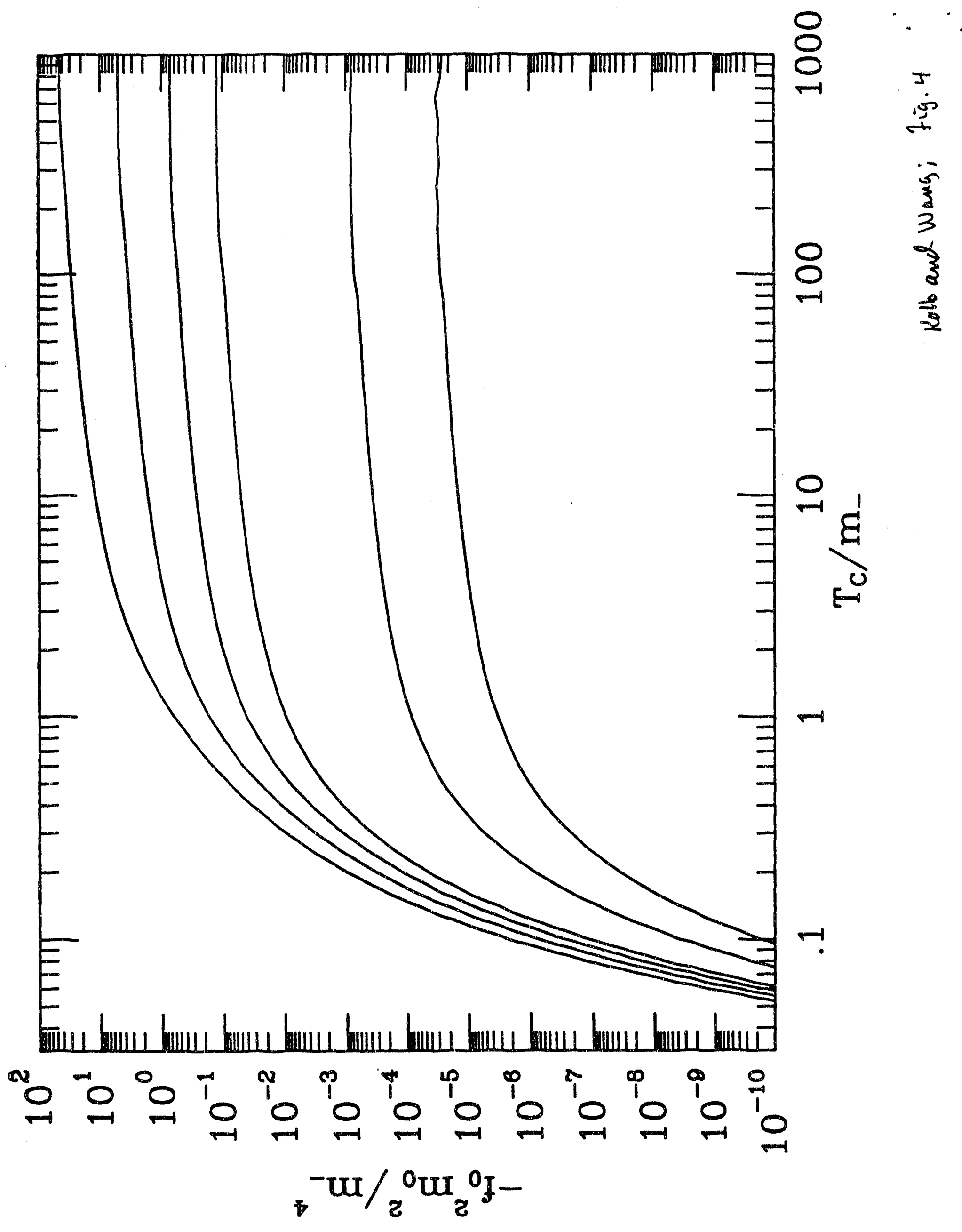




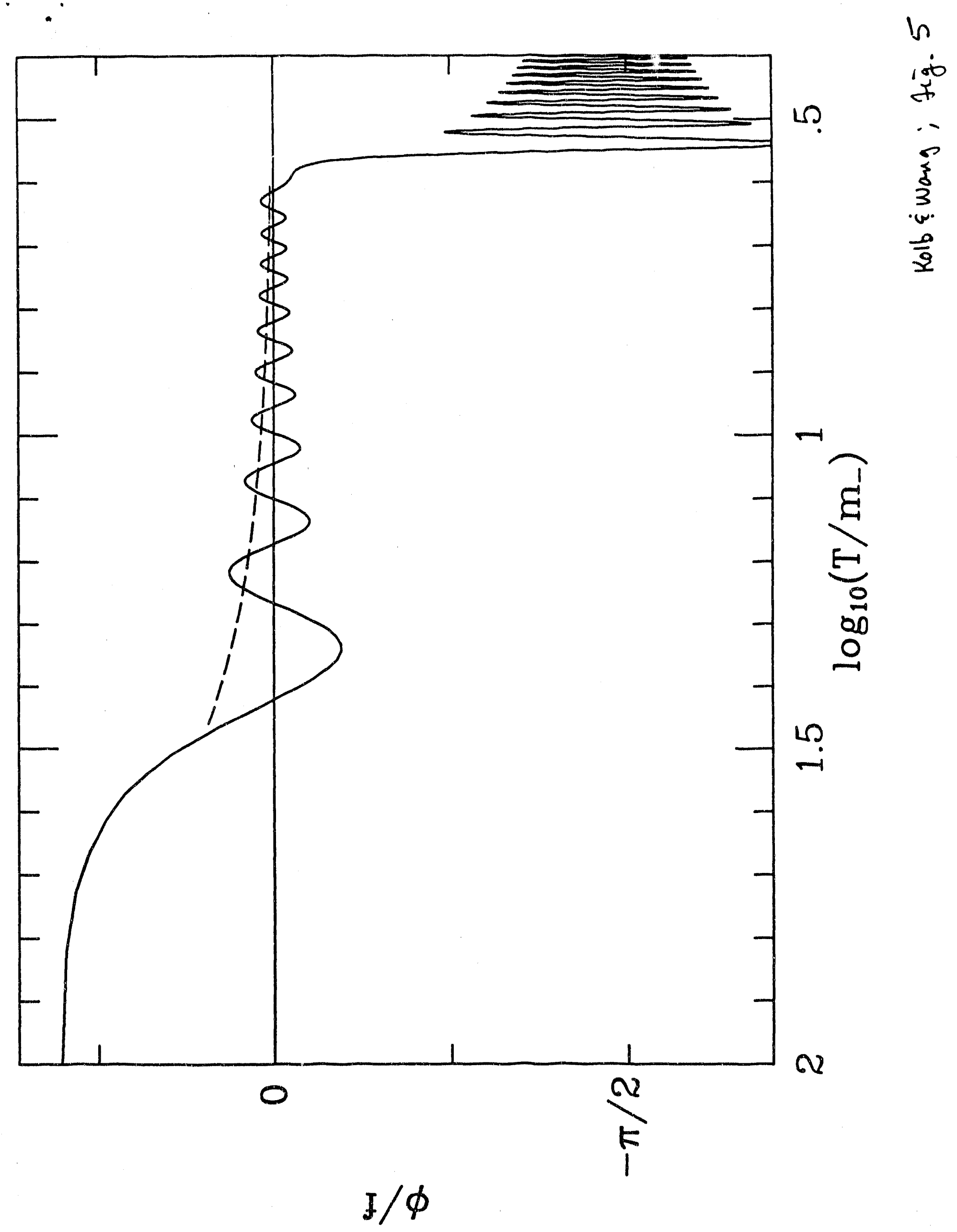



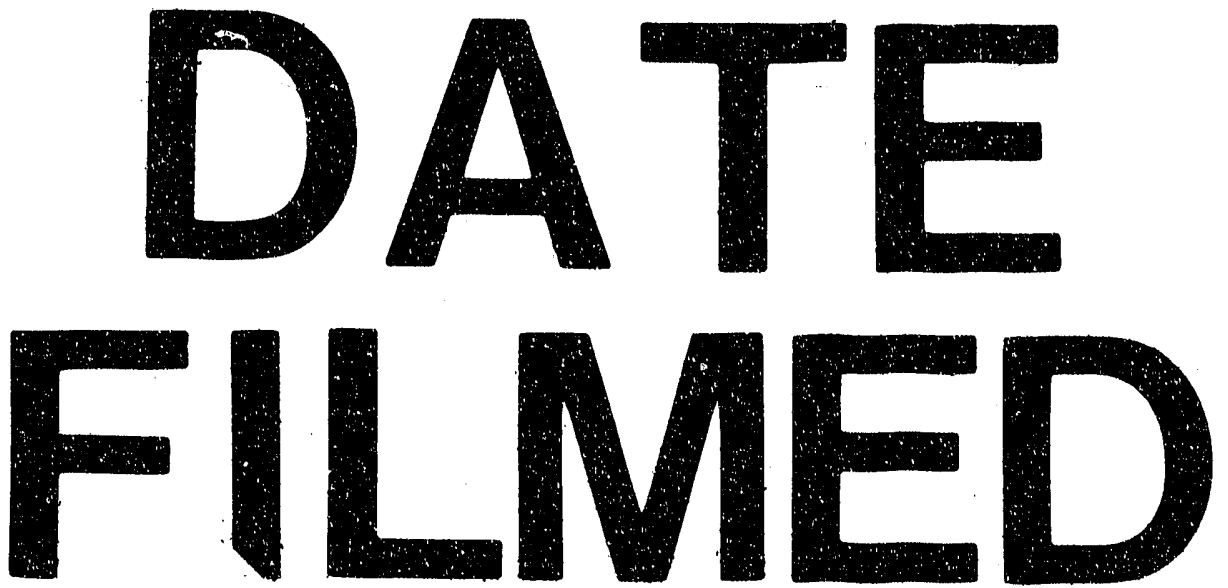

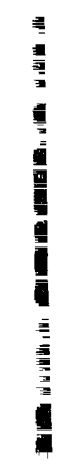

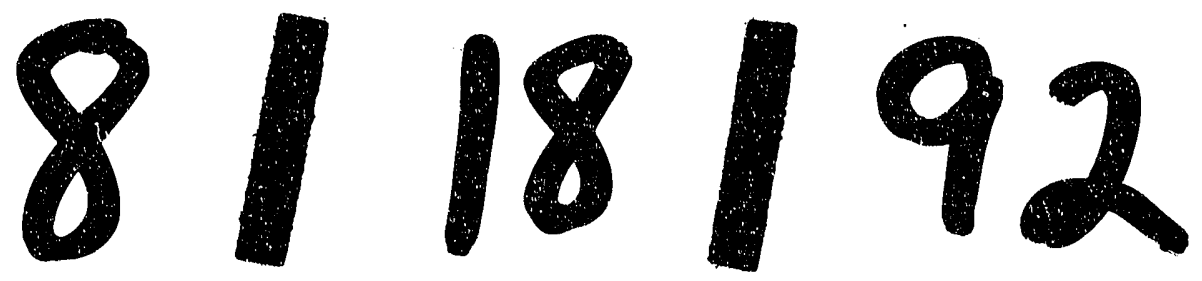


